\title{
Comparative Performance of Turnip (Brassica rapa var. rapifera L.) Genotypes for Various Quantitative and Quality Traits
}

\author{
Mir Tabasum Ashraf*, Shahnaz Mufti , Khursheed Hussain, Gowhar Ali, Usma jan, \\ Nawaz Ahmad, Iqtab Mushtaq, Afiya Khursheed, Humeera Nazir, Sameena Maqbool \\ and Amreena Sultan
}

\author{
Division of Vegetable Science SKUAST-Shalimar Kashmir, India \\ *Corresponding author
}

\begin{abstract}
A B S T R A C T
Keywords

Comparative

Performance,

Brassica rapa var.

rapifera $\mathrm{L}$.,

Genotypes,

Variability

Article Info

Accepted:

12 December 2020

Available Online:

10 January 2021

The present investigation entitled Comparative Performance of Turnip (Brassica rapa var. rapifera L.) genotypes for various quantitative and quality traits was carried out at Vegetable Experimental Field, Division of Vegetable Science, SKUAST-K Shalimar, Kashmir to evaluate the mean performance of twenty-eight turnip genotypes during Rabi 2109. The experiment was carried out in Randomized complete block design (RCBD) with three replications for each genotype and the crop was sown at a spacing of $30 \times 15 \mathrm{~cm}$. The analysis of variance revealed mean sum of squares due to genotypes were significant for all the traits that were studied, indicating the presence of sufficient variability among the genotypes. Based on the mean performance of all the genotypes for various traits studied, the genotype viz., SKAU-T-4 was at par with Nageen which showed highest root yield followed by SKAU-T-3 while as minimum root yield was recorded in SKAU-T-23.
\end{abstract}

\section{Introduction}

Brassica is a diverse genus that contains species used for oilseeds, leafy or root vegetables, and condiments (Persson et al., 2001; Talebi et al., 2010). One important food crop is turnip (Brassica rapa var. rapifera L.) $(2 \mathrm{n}=2 \mathrm{x}=20)$ which is a biennial root vegetable cultivated worldwide as vegetable and fodder (Rakow, 2004; Hammer et al., 2013). It is said to have two centres of origin. The Mediterranean region is thought to be the primary centre of European types while as
Eastern Afghanistan with adjoining area of Pakistan is considered to be another primary centre. It is an important root vegetable grown as a summer crop in temperate climate and as a winter vegetable in subtropical places where the winter is not severe. It can be grown up to an elevation of $1500 \mathrm{~m}$ above mean sea level or above but it is not suitable for growing in low lands of wet tropics (Thamburaj and Singh 2018). In India it is cultivated in an area of 2500 ha with an annual production of 50,000 tonnes (Anonymous, 2017). 
The economic part is the thickened underground portion which is actually hypocotyl. The colour of this underground portion may be white or yellow while as that of the aboveground portion may be red, purple, yellow, pink or green. Normally the roots attain edible maturity in 40-80 days after sowing depending upon the cultivar and cultural conditions. Brassica flowers are born in racemes and are hermaphrodite but due to self-incompatibility these are cross pollinated.

The flowers open during the early hours of daylight and few hours later the anthers dehisce exposing their pollen while as the stigma becomes receptive five days prior to anthesis and remains up to four days after anthesis and during this time cross pollination is obligatory (McCormack, 2005)

There is scanty of literature regarding performance of turnip genotypes. So present study will generate the extent of variation among different genotypes of turnip for various quantitative and quality traits.

\section{Materials and Methods}

The present investigation was carried out at Vegetable Experimental field, SKAUST-K, Shalimar during Rabi 2019. The site of experimental field is located at an altitude of 1685 meters above mean sea level and situated at $34^{0} \mathrm{~N}$ latitude and $74.89^{\circ} \mathrm{E}$ longitude. The climate of the site is temperate characterized by mild summers. The mean maximum and minimum temperatures are recorded in June-July and January-February (respectively).

Twenty-eight genotypes of turnip were evaluated for various quantitative and quality traits. The experiment was carried out in Randomized block design with three replications for each genotype. The crop was sown at a spacing of $30 \mathrm{~cm}$ row to row distance and $15 \mathrm{~cm}$ plant to plant distance. The crop was grown according to recommended package of practices.

The observations were recorded for nineteen quantitative traits viz., seedling length, seedling fresh weight, seedling dry weight, leaf length, leaf breadth, petiole length, root length, root breadth, plant length, leaves/plant, total plant weight, total root weight, total shoot weight, root shoot ratio, root compactness, harvest index, root yield/ha, plant spread and leaf area and seven quality traits viz., total dry matter, total soluble solids, vitamin $\mathrm{C}$, anthocyanin content, total sugars, reducing and nonreducing sugars.

Observations were recorded on five randomly selected plants of each genotype from all the three replications and mean was worked out. The data was subjected to statistical analysis using WINDOSTAT software package.

The data of quantitative and quality traits was statistically analysed as per the method lined by Gomez and Gomez (1983) for estimating the analysis of variance.

\section{Results and Discussion}

\section{Analysis of variance}

Analysis of variance (ANOVA) exposed significant differences among all the genotypes for all the traits that were studied during present investigation (Table 1a and Table 1b) hence, depicting the presence of adequate genetic variability among these turnip genotypes and substantial scope of improvement for these traits.

This was in agreement with Nasarullah (1994) in turnip, Mukhdoomi et al., (2007) in radish, Naseeruddin et al., (2011) in radish, Guleria (2016) in radish. 
Table.1a Analysis of variance for various quantitative traits in Turnip (Brassica rapa var. rapifera L.).

\begin{tabular}{|c|c|c|c|c|c|c|c|c|c|c|c|c|c|c|c|c|c|c|c|c|}
\hline \multirow[t]{2}{*}{$\begin{array}{l}\text { Source of } \\
\text { variation }\end{array}$} & \multirow[b]{2}{*}{ D.f } & \multicolumn{19}{|c|}{ Mean sum of squares } \\
\hline & & $\begin{array}{l}\text { Seedlin } \\
\text { g length } \\
\text { (cm) }\end{array}$ & $\begin{array}{l}\text { Seedlin } \\
\text { g fresh } \\
\text { weight } \\
\text { (g) }\end{array}$ & $\begin{array}{l}\text { Seedli } \\
\text { ng dry } \\
\text { weight } \\
\text { (g) }\end{array}$ & $\begin{array}{l}\text { Leaf } \\
\text { lengt } \\
h \\
(\mathrm{~cm})\end{array}$ & $\begin{array}{l}\text { Leaf } \\
\text { breadt } \\
\text { h } \\
\text { (cm) }\end{array}$ & $\begin{array}{l}\text { Petio } \\
\text { le } \\
\text { lengt } \\
\text { h } \\
\text { (cm) }\end{array}$ & $\begin{array}{l}\text { Root } \\
\text { lengt } \\
\text { h } \\
(\mathrm{cm})\end{array}$ & $\begin{array}{l}\text { Root } \\
\text { brea } \\
\text { dth } \\
(\mathbf{c m})\end{array}$ & $\begin{array}{l}\text { Plant } \\
\text { length } \\
(\mathrm{cm})\end{array}$ & $\begin{array}{l}\text { Leaves } \\
\text { per } \\
\text { plant }\end{array}$ & $\begin{array}{l}\text { Total } \\
\text { plant } \\
\text { weight } \\
\text { (g) }\end{array}$ & $\begin{array}{l}\text { Total } \\
\text { root } \\
\text { weight } \\
\text { (g) }\end{array}$ & $\begin{array}{l}\text { Total } \\
\text { shoot } \\
\text { weight } \\
\text { (g) }\end{array}$ & $\begin{array}{l}\text { Root } \\
\text { shoo } \\
\text { t } \\
\text { ratio }\end{array}$ & $\begin{array}{l}\text { Root } \\
\text { compa } \\
\text { ctness }\end{array}$ & $\begin{array}{l}\text { Harves } \\
t \text { index } \\
(\%)\end{array}$ & $\begin{array}{l}\text { Root } \\
\text { yield } \\
\text { (q/ha) }\end{array}$ & $\begin{array}{l}\text { Plant } \\
\text { spread } \\
\text { (cm) }\end{array}$ & $\begin{array}{l}\text { Leaf } \\
\text { area } \\
\left(\mathrm{cm}^{2}\right)\end{array}$ \\
\hline Replication & 2 & $10.56^{*}$ & 0.09 & $0.003 *$ & $\begin{array}{l}11.76 \\
*\end{array}$ & $\begin{array}{l}\text { 28.36* } \\
*\end{array}$ & 3.04 & $\begin{array}{l}\mathbf{0 . 7 2} \\
*\end{array}$ & 0.08 & 10.04 & $\begin{array}{l}16.94 * \\
*\end{array}$ & 11.67 & 38.61 & 14.59 & 0.15 & 277.27 & 22.78* & 144.083 & 5.59 & 8.49 \\
\hline Treatments & 27 & $11.28 * *$ & $1.64 * *$ & $0.04 * *$ & $\begin{array}{l}10.41 \\
* *\end{array}$ & $6.16^{* * *}$ & $\begin{array}{l}\mathbf{8 . 3 3} \\
* *\end{array}$ & $\begin{array}{l}\mathbf{0 . 8 6} \\
* *\end{array}$ & $\begin{array}{l}3.15 \\
* *\end{array}$ & $\begin{array}{l}15.12 * \\
*\end{array}$ & $1.54 * *$ & $\begin{array}{l}7405.1 \\
2 *\end{array}$ & $\begin{array}{l}4872.5 \\
4^{*}\end{array}$ & $\begin{array}{l}\mathbf{3 4 2 . 3 8} \\
* *\end{array}$ & $\begin{array}{l}\mathbf{0 . 8 4} \\
* *\end{array}$ & $\begin{array}{l}\mathbf{7 5 3 . 4 4} \\
* *\end{array}$ & $\begin{array}{l}\text { 25.35* } \\
*\end{array}$ & $\begin{array}{l}19439.75 \\
* *\end{array}$ & $\begin{array}{l}\text { 32.08* } \\
*\end{array}$ & $\begin{array}{l}\text { 2013.92* } \\
*\end{array}$ \\
\hline error & 54 & 1.88 & 0.20 & 0.0008 & 2.13 & 1.24 & 2.15 & 0.15 & 0.15 & 3.89 & 0.59 & 62.35 & 14.11 & 13.28 & 0.08 & 95.77 & 6.59 & 55.09 & 4.77 & 10.76 \\
\hline
\end{tabular}

Table.1b Analysis of variance for various quality traits in Turnip (Brassica rapa var. rapifera L.).

\begin{tabular}{|c|c|c|c|c|c|c|c|c|}
\hline \multirow[b]{2}{*}{ Source of variation } & \multirow[b]{2}{*}{ d. $f$} & \multicolumn{7}{|c|}{ Mean sum of squares } \\
\hline & & $\begin{array}{l}\text { Total dry matter } \\
(\%)\end{array}$ & $\begin{array}{l}\text { Total soluble } \\
\text { Solids ( }{ }^{\circ} \text { Brix }\end{array}$ & $\begin{array}{l}\text { Vit. C } \\
\text { (mg/100g) }\end{array}$ & $\begin{array}{l}\text { Anthocyanin } \\
\text { Content (mg/100g) }\end{array}$ & $\begin{array}{l}\text { Total sugars } \\
(\%)\end{array}$ & $\begin{array}{l}\text { Reducing } \\
\text { Sugars (\%) }\end{array}$ & $\begin{array}{l}\text { Non- reducing } \\
\text { Sugars }(\%)\end{array}$ \\
\hline Replication & 2 & 1.01 & 0.15 & 8.03 & 0.004 & 0.11 & 0.09 & 0.13 \\
\hline Treatments & 27 & $8.50 * *$ & $2.41 * *$ & $52.38 * *$ & $3.33 * *$ & $0.99 * *$ & $0.37 * *$ & $0.23 * *$ \\
\hline Error & 54 & 2.46 & 0.07 & 5.67 & 0.03 & 0.09 & 0.03 & 0.06 \\
\hline
\end{tabular}

$*, * *$ - Significant at $5 \%$ and $1 \%$ level of significance respectively 
Table.2a Mean performance of Turnip (Brassica rapa var. rapifera) genotypes for various quantitative traits

\begin{tabular}{|c|c|c|c|c|c|c|c|c|c|c|c|}
\hline $\begin{array}{l}\text { S. } \\
\text { No }\end{array}$ & Genotype & $\begin{array}{l}\text { Seedling } \\
\text { length } \\
\text { (cm) }\end{array}$ & $\begin{array}{l}\text { Seedling } \\
\text { fresh weight } \\
\text { (g) }\end{array}$ & $\begin{array}{l}\text { Seedling } \\
\text { dry } \\
\text { weight } \\
\text { (g) }\end{array}$ & $\begin{array}{l}\text { Leaf length } \\
\text { (cm) }\end{array}$ & $\begin{array}{l}\text { Leaf breadth } \\
\text { (cm) }\end{array}$ & $\begin{array}{l}\text { Petiole length } \\
\text { (cm) }\end{array}$ & $\begin{array}{l}\text { Root length } \\
\text { (cm) }\end{array}$ & $\begin{array}{l}\text { Root } \\
\text { breadth } \\
\text { (cm) }\end{array}$ & $\begin{array}{l}\text { Plant length } \\
\text { (cm) }\end{array}$ & $\begin{array}{l}\text { Leaves } \\
\text { plant }^{-1}\end{array}$ \\
\hline 1 & SKAU-T-1 & $22.90^{\text {cdefgh }}$ & $5.13^{\mathrm{abcd}}$ & $0.50^{\mathrm{efg}}$ & $18.70^{\text {h }}$ & $11.93^{\text {bcdetgh }}$ & $12.36^{\text {ghijk }}$ & $4.91^{\text {ghij }}$ & $6.33^{\text {def }}$ & $51.16^{\text {efghij }}$ & $9.00^{\mathrm{ef}}$ \\
\hline 3 & SKAU-T-3 & $18.73^{k}$ & $3.53^{\operatorname{Imnop}}$ & $0.30^{\mathbf{n}}$ & $21.30^{\text {cdefg }}$ & $12.46^{\text {bcdefg }}$ & $10.96^{k}$ & $5.83^{\mathrm{bcd}}$ & $7.76^{\mathrm{ab}}$ & $55.23^{\mathrm{abc}}$ & $9.33^{\text {def }}$ \\
\hline 4 & SKAU-T-4 & $21.66^{\text {elghij }}$ & $3.87^{\mathrm{ijk} k \mathrm{mn}}$ & $0.42^{i j k}$ & $23.30^{\text {abc }}$ & $11.23^{\text {etgh }}$ & $14.14^{\text {bcdetgh }}$ & $5.20^{\text {defghi }}$ & $7.20^{\mathrm{bc}}$ & $55.60^{\mathrm{ab}}$ & $11.00^{\mathrm{ab}}$ \\
\hline 5 & Nageen & $21.16^{\text {ghij }}$ & $3.10^{\mathrm{op}}$ & $0.30^{n}$ & $24.43^{\mathrm{a}}$ & $16.30^{\mathrm{a}}$ & $14.80^{\text {abcdef }}$ & $5.60^{\text {bcdef }}$ & $7.10^{c}$ & $52.96^{\text {bcdefg }}$ & $9.00^{\text {ef }}$ \\
\hline 6 & SKAU-T-5 & $20.83^{\text {hijk }}$ & $3.84^{\mathrm{jklmno}}$ & $0.45^{\mathrm{hij}}$ & $22.90^{\text {abcd }}$ & $13.10^{\text {bcd }}$ & $13.05^{\text {defghijk }}$ & $5.26^{\text {cdefg }}$ & $7.76^{\mathbf{a b}}$ & $51.40^{\text {defghij }}$ & $9.66^{\text {cdef }}$ \\
\hline 8 & SKAU-T-7 & $19.66^{\mathrm{jk}}$ & $3.37^{\mathrm{mnop}}$ & $0.35^{\mathrm{lm}}$ & $21.70^{\text {bcdef }}$ & $12.30^{\text {bcdefgh }}$ & $17.05^{\mathrm{a}}$ & $5.60^{\text {bcdef }}$ & $7.93^{\mathrm{a}}$ & $56.06^{\mathrm{ab}}$ & $10.66^{\mathrm{abc}}$ \\
\hline 9 & SKAU-T-8 & $21.83^{\text {efghij }}$ & $2.95^{\mathrm{P}}$ & $0.41^{\mathbf{j k}}$ & $20.40^{\text {efgh }}$ & $10.60^{\text {hij }}$ & $11.40^{\mathrm{ijk}}$ & $4.96^{\text {fghij }}$ & $7.10^{c}$ & $54.23^{\text {abcde }}$ & $10.33^{\text {abcd }}$ \\
\hline 10 & SKAU-T-9 & $21.33^{\text {fghij }}$ & $4.16^{\text {fghijkl }}$ & $0.52^{\mathrm{ef}}$ & $22.13^{\text {abcde }}$ & $11.30^{\text {defgh }}$ & $14.96^{\text {abcdef }}$ & $5.00^{\text {efghi }}$ & $7.20^{\mathbf{b c}}$ & $54.40^{\text {abcd }}$ & $9.33^{\text {def }}$ \\
\hline 11 & SKAU-T-10 & $23.90^{\text {bcde }}$ & $4.70^{\text {cdefgh }}$ & $0.57^{\text {cd }}$ & $20.83^{\text {defgh }}$ & $12.00^{\text {bcdefgh }}$ & $12.93^{\text {defghijk }}$ & $4.56^{\mathrm{ijk}}$ & $6.96^{\mathrm{cd}}$ & $51.13^{\text {efghij }}$ & $9.00^{\text {ef }}$ \\
\hline 12 & SKAU-T-11 & $21.10^{\text {ghij }}$ & $3.73^{\mathrm{klmno}}$ & $0.43^{\mathrm{ij}}$ & $21.16^{\text {cdefg }}$ & $12.50^{\text {bcdefg }}$ & $13.50^{\text {cdefghij }}$ & $5.26^{\text {cdefg }}$ & $6.76^{\text {cde }}$ & $50.73^{\text {fghij }}$ & $9.33^{\text {def }}$ \\
\hline 13 & PTWG & $23.53^{\text {cdef }}$ & $4.30^{\text {efghijk }}$ & $0.52^{\mathrm{ef}}$ & $22.80^{\text {abcd }}$ & $12.60^{\text {bcdef }}$ & $10.96^{\mathrm{k}}$ & $4.33^{\mathbf{j k}}$ & $7.36^{\mathrm{abc}}$ & $53.33^{\text {abcdefg }}$ & $10.00^{\text {bcde }}$ \\
\hline 14 & SKAU-T-12 & $21.73^{\text {efghij }}$ & $3.96^{\mathrm{hijklm}}$ & $0.51^{\text {efg }}$ & $19.76^{\text {efgh }}$ & $10.60^{\text {hij }}$ & $12.70^{\text {efghijk }}$ & $5.23^{\text {defgh }}$ & $7.13^{\mathrm{bc}}$ & $49.20^{\text {hij }}$ & $10.00^{\text {bcde }}$ \\
\hline 15 & SKAU-T-13 & $23.13^{\text {cdefg }}$ & $4.83^{\text {bcdef }}$ & $0.55^{\mathrm{de}}$ & $23.33^{\mathrm{abc}}$ & $12.80^{\text {bcde }}$ & $12.70^{\text {efghijk }}$ & $4.23^{\mathrm{k}}$ & $7.26^{\mathrm{bc}}$ & $48.60^{j}$ & $9.00^{\text {ef }}$ \\
\hline 16 & SKAU-T-14 & $22.03^{\text {detghi }}$ & $4.53^{\text {defghij }}$ & $0.50^{\text {efg }}$ & $22.90^{\text {abcd }}$ & $12.76^{\text {bcde }}$ & $15.46^{\mathrm{abc}}$ & $5.73^{\mathrm{bcd}}$ & $7.20^{\mathbf{b c}}$ & $56.43^{\mathrm{a}}$ & $9.00^{\text {ef }}$ \\
\hline 19 & SKAU-T-17 & $25.83^{\text {ab }}$ & $4.73^{\text {cdefg }}$ & $0.60^{\mathbf{c}}$ & $20.70^{\text {defgh }}$ & $11.26^{\text {efgh }}$ & $13.43^{\text {cdefghij }}$ & $5.26^{\text {cdefg }}$ & $5.13^{\mathrm{hi}}$ & $48.76^{\mathrm{ij}}$ & $9.33^{\text {def }}$ \\
\hline 20 & SKAU-T-18 & $23.16^{\text {cdefg }}$ & $4.60^{\text {cdefghi }}$ & $0.60^{\mathrm{c}}$ & $21.06^{\text {cdefgh }}$ & $12.36^{\text {bcdefgh }}$ & $11.76^{\text {hijk }}$ & $6.66^{\mathrm{a}}$ & $4.73^{\mathrm{i}}$ & $54.33^{\text {abcde }}$ & $10.33^{\text {abcd }}$ \\
\hline 21 & SKAU-T-19 & $22.73^{\text {cdefgh }}$ & $4.11^{\text {fghijklm }}$ & $0.49^{\text {figh }}$ & $19.22^{\text {gh }}$ & $11.63^{\text {cdefgh }}$ & $15.00^{\text {abcde }}$ & $4.71^{\text {ghijk }}$ & $6.06^{\mathrm{fg}}$ & $53.80^{\text {abcdef }}$ & $10.66^{\text {abc }}$ \\
\hline 22 & SKAU-T-20 & $24.46^{\mathrm{bc}}$ & $5.05^{\text {abcd }}$ & $0.66^{\mathrm{ab}}$ & $19.33^{\text {fgh }}$ & $11.23^{\mathrm{efgh}}$ & $15.30^{\text {abcd }}$ & $5.96^{b}$ & $6.23^{\mathrm{efg}}$ & $51.20^{\text {defghij }}$ & $11.33^{\mathrm{a}}$ \\
\hline 23 & SKAU-T-21 & $22.76^{\text {cdefgh }}$ & $5.28^{\mathrm{abc}}$ & $0.66^{b}$ & $21.73^{\text {bcde }}$ & $10.93^{\text {fghi }}$ & $15.33^{\text {abcd }}$ & $4.73^{\text {ghijk }}$ & $5.16^{\mathrm{hi}}$ & $51.30^{\text {defghij }}$ & $10.00^{\text {bcde }}$ \\
\hline 24 & SKAU-T-22 & $26.06^{\mathrm{ab}}$ & $5.12^{\text {abcd }}$ & $0.59^{\text {cd }}$ & $20.36^{\text {efgh }}$ & $8.86^{\mathbf{j}}$ & $12.06^{\text {hijk }}$ & $4.80^{\text {ghijk }}$ & $4.83^{\mathrm{i}}$ & $50.23^{\text {ghij }}$ & $10.33^{\text {abcd }}$ \\
\hline 25 & SKAU-T-23 & $24.66^{\mathrm{bc}}$ & $4.82^{\text {bcdef }}$ & $0.50^{\text {fgh }}$ & $16.00^{i}$ & $9.13^{\mathrm{ij}}$ & $16.40^{\mathrm{ab}}$ & $5.00^{\text {efghi }}$ & $4.83^{i}$ & $49.43^{\text {hij }}$ & $9.33^{\text {def }}$ \\
\hline 26 & SKAU-T-24 & $27.23^{\mathrm{a}}$ & $5.71^{\mathrm{a}}$ & $0.71^{\mathrm{a}}$ & $18.70^{\mathrm{h}}$ & $10.70^{\text {ghi }}$ & $15.13^{\mathrm{abcd}}$ & $5.20^{\text {defghi }}$ & $5.23^{\mathrm{hi}}$ & $53.00^{\text {bcdefg }}$ & $8.66^{f}$ \\
\hline 27 & SKAU-T-25 & $23.90^{\text {bcde }}$ & $4.29^{\text {efghijk }}$ & $0.38^{\mathbf{k} \mathbf{I}}$ & $21.63^{\text {bcdef }}$ & $12.36^{\text {bcdefgh }}$ & $12.26^{\text {ghijk }}$ & $4.60^{\mathrm{hijk}}$ & $4.76^{\mathrm{i}}$ & $51.83^{\text {defghi }}$ & $9.33^{\text {def }}$ \\
\hline 28 & SKAU-T-26 & $23.33^{\text {cdefg }}$ & $4.05^{\text {fghijkl }}$ & $0.46^{\mathrm{ghi}}$ & $23.00^{\text {abcd }}$ & $12.00^{\text {bcdefgh }}$ & $12.56^{\text {fghijk }}$ & $5.33^{\text {bcdefg }}$ & $5.63^{\text {gh }}$ & $52.03^{\text {cdefgh }}$ & $10.33^{\text {abcd }}$ \\
\hline \multicolumn{2}{|c|}{ C.D.5\% } & 2.24 & 0.74 & 0.04 & 2.38 & 1.82 & 2.40 & 0.64 & 0.63 & 3.23 & 1.26 \\
\hline \multicolumn{2}{|c|}{ S.E.Diff } & 1.12 & 0.37 & 0.02 & 1.19 & 0.91 & 1.19 & 0.32 & 0.31 & 1.61 & 0.62 \\
\hline
\end{tabular}


Table.2b Mean performance of Turnip (Brassica rapa var. rapifera) genotypes for various quantitative traits

\begin{tabular}{|c|c|c|c|c|c|c|c|c|c|c|}
\hline S.no & Genotype & $\begin{array}{l}\text { Total plant } \\
\text { weight }(g)\end{array}$ & $\begin{array}{l}\text { Total root } \\
\text { weight(g) }\end{array}$ & $\begin{array}{l}\text { Total shoot } \\
\text { weight(g) }\end{array}$ & $\begin{array}{l}\text { Root shoot } \\
\text { ratio }\end{array}$ & $\begin{array}{l}\text { Root } \\
\text { compactness }\end{array}$ & $\begin{array}{l}\text { Harvest } \\
\text { index }(\%)\end{array}$ & $\begin{array}{l}\text { Root yield } \\
\text { (q/ha)) }\end{array}$ & $\begin{array}{l}\text { Plant spread } \\
\text { (cm) }\end{array}$ & $\begin{array}{l}\text { Leaf area } \\
\left(\mathrm{cm}^{2}\right)\end{array}$ \\
\hline 1 & SKAU-T-1 & $173.33^{\text {hij }}$ & $134.66^{\mathrm{h}}$ & $40.00^{\text {hijk }}$ & $3.36^{\text {defgh }}$ & $76.78^{\text {bcdefg }}$ & $77.70^{\text {bcdef }}$ & $269.33^{h}$ & $52.00^{\text {cdefgh }}$ & $227.70^{\mathrm{fg}}$ \\
\hline 2 & SKAU-T-2 & $194.66^{\mathrm{fg}}$ & $145.33^{\mathrm{g}}$ & $46.66^{\text {efg }}$ & $3.13^{\text {ghi }}$ & $62.90^{\text {fghij }}$ & $74.60^{\text {efgh }}$ & $290.66^{\mathrm{g}}$ & $51.60^{\text {cdefghi }}$ & $259.40^{\mathbf{c}}$ \\
\hline 3 & SKAU-T-3 & $260.66^{b}$ & $201.16^{b}$ & $61.83^{\mathbf{a b}}$ & $3.22^{\text {fghi }}$ & $63.66^{\text {efghij }}$ & $76.50^{\text {cdef }}$ & $402.33^{b}$ & $50.46^{\text {defghij }}$ & $204.53^{\mathrm{m}}$ \\
\hline 4 & SKAU-T-4 & $285.00^{\mathrm{a}}$ & $231.00^{\mathrm{a}}$ & $54.00^{\text {cd }}$ & $4.29^{\mathrm{a}}$ & $91.00^{\mathbf{a b}}$ & $81.03^{\mathbf{a b}}$ & $462.00^{\mathrm{a}}$ & $47.40^{\mathbf{j k}}$ & $221.93^{\mathrm{hi}}$ \\
\hline 5 & Nageen & $295.00^{\mathrm{a}}$ & $235.33^{\mathrm{a}}$ & $56.00^{b c}$ & $4.17^{\mathrm{ab}}$ & $91.46^{\mathbf{a b}}$ & $79.01^{\text {abcd }}$ & $470.66^{a}$ & $50.46^{\text {defghij }}$ & $262.33^{b c}$ \\
\hline 6 & SKAU-T-5 & $144.66^{\mathrm{mn}}$ & $107.00^{\mathrm{kl}}$ & $37.66^{\mathrm{ijkl}}$ & $2.84^{\mathrm{ij}}$ & $39.24^{1}$ & $73.96^{\text {fghi }}$ & $214.00^{\mathrm{kI}}$ & $54.76^{\mathrm{bc}}$ & $266.46^{b}$ \\
\hline 7 & SKAU-T-6 & $214.33^{d}$ & $165.66^{e}$ & $50.00^{\text {def }}$ & $3.35^{\text {defgh }}$ & $72.51^{\text {defgh }}$ & $77.46^{\text {bcdef }}$ & $331.33^{\mathrm{e}}$ & $53.16^{\text {cdefg }}$ & $287.63^{a}$ \\
\hline 8 & SKAU-T-7 & $209.66^{\text {de }}$ & $166.00^{\mathrm{e}}$ & $46.33^{\text {efg }}$ & $3.59^{\text {cdefg }}$ & $53.74^{\mathrm{ijkl}}$ & $79.28^{\mathrm{abc}}$ & $332.00^{\mathrm{e}}$ & $58.30^{\mathbf{a b}}$ & $206.36^{\mathrm{lm}}$ \\
\hline 9 & SKAU-T-8 & $209.33^{\text {de }}$ & $170.66^{\mathrm{de}}$ & $45.33^{\text {efgh }}$ & $3.73^{\text {bcde }}$ & $77.54^{\text {bcdef }}$ & $77.06^{\text {bcdef }}$ & $341.33^{\text {de }}$ & $51.56^{\text {cdefghi }}$ & $217.43^{i j}$ \\
\hline 10 & SKAU-T-9 & $271.00^{b}$ & $200.00^{b}$ & $64.66^{\mathrm{a}}$ & $3.00^{h i j}$ & $87.50^{\text {abcd }}$ & $73.90^{\text {fghi }}$ & $400.00^{b}$ & $51.60^{\text {cdefghi }}$ & $252.30^{d}$ \\
\hline 11 & SKAU-T-10 & $164.00^{\mathbf{j k}}$ & $126.00^{i}$ & $53.33^{\mathrm{cd}}$ & $2.20^{1}$ & $61.16^{\text {ghijk }}$ & $71.40^{\mathrm{hi}}$ & $252.00^{\mathrm{i}}$ & $50.26^{\text {efghij }}$ & $183.26^{\mathbf{p}}$ \\
\hline 12 & SKAU-T-11 & $149.33^{\mathrm{Im}}$ & $108.00^{\mathrm{kl}}$ & $41.33^{\text {ghij }}$ & $2.63^{j k l}$ & $49.66^{\mathrm{jkl}}$ & $72.26^{\text {ghi }}$ & $216.00^{\mathrm{kI}}$ & $50.93^{\text {defghij }}$ & $214.76^{j k}$ \\
\hline 13 & PTWG & $228.33^{c}$ & $176.33^{\text {cd }}$ & $62.66^{\mathrm{a}}$ & $2.81^{\mathrm{ijk}}$ & $89.65^{a b c}$ & $77.26^{\text {bcdef }}$ & $352.66^{\text {cd }}$ & $54.00^{\text {cd }}$ & $195.96^{\text {no }}$ \\
\hline 14 & SKAU-T-12 & $140.66^{\mathrm{mn}}$ & $108.00^{\mathbf{j k}}$ & $32.66^{\operatorname{lm}}$ & $3.33^{\text {defgh }}$ & 46.23 & $76.70^{\text {cdef }}$ & $219.33^{\mathbf{j k}}$ & $58.86^{\mathrm{a}}$ & $203.83^{\mathbf{m}}$ \\
\hline 15 & SKAU-T-13 & $165.33^{\mathrm{ijk}}$ & $124.00^{i}$ & $41.33^{\text {ghij }}$ & $2.99^{h i j}$ & $65.93^{\text {efghi }}$ & $74.93^{\text {defgh }}$ & $248.00^{i}$ & $51.33^{\text {cdefghi }}$ & $217.06^{\mathbf{i j}}$ \\
\hline 16 & SKAU-T-14 & $218.66^{\text {cd }}$ & $181.33^{c}$ & $44.33^{\text {fgh }}$ & $4.12^{\text {ab }}$ & $67.09^{\text {efghi }}$ & $82.96^{\mathrm{a}}$ & $362.66^{\mathrm{c}}$ & $46.50^{k}$ & $220.20^{\mathrm{hi}}$ \\
\hline 17 & SKAU-T-15 & $163.33^{j k}$ & $127.33^{i}$ & $41.33^{\text {ghij }}$ & $3.02^{h i j}$ & $46.46^{\mathrm{kl}}$ & $76.33^{\text {cdefg }}$ & $254.66^{i}$ & $51.13^{\text {defghi }}$ & $240.20^{\mathrm{e}}$ \\
\hline 18 & SKAU-T-16 & $136.66^{\mathrm{mn}}$ & $108.00^{\mathrm{kl}}$ & $34.33^{\mathrm{klm}}$ & $3.15^{\mathrm{ghi}}$ & $58.76^{\text {hijk }}$ & $79.05^{\text {abcd }}$ & $216.00^{\mathrm{kI}}$ & $46.50^{k}$ & $218.33^{\mathrm{hi}}$ \\
\hline 19 & SKAU-T-17 & $159.33^{\mathrm{kI}}$ & $126.00^{i}$ & $34.00^{\mathrm{lm}}$ & $3.69^{\text {bcdef }}$ & $86.63^{\text {abcd }}$ & $78.60^{\text {bcde }}$ & $252.00^{\mathrm{i}}$ & $53.73^{\text {cdef }}$ & $223.60^{\text {gh }}$ \\
\hline 20 & SKAU-T-18 & $145.33^{\mathrm{mn}}$ & $102.00^{1}$ & $43.33^{\text {ghi }}$ & $2.35^{\mathrm{kl}}$ & $55.66^{\mathrm{ijk}}$ & $70.16^{i}$ & $204.00^{1}$ & $49.86^{\text {ghijk }}$ & $219.83^{\text {hij }}$ \\
\hline 21 & SKAU-T-19 & $199.66^{\text {ef }}$ & $151.66^{f}$ & $50.33^{\text {cde }}$ & $2.97^{h i j}$ & $95.30^{\mathbf{a}}$ & $74.73^{\text {efgh }}$ & $303.33^{f}$ & $53.50^{\text {cdef }}$ & $252.76^{d}$ \\
\hline 22 & SKAU-T-20 & $178.00^{\mathrm{hi}}$ & $144.33^{\mathrm{g}}$ & $36.66^{\mathrm{jklm}}$ & $3.85^{\mathrm{abc}}$ & $62.70^{\text {fghij }}$ & $79.38^{\text {abc }}$ & $288.66^{\mathrm{g}}$ & $53.06^{\text {cdefg }}$ & $211.36^{\mathrm{kl}}$ \\
\hline 23 & SKAU-T-21 & $145.33^{\mathrm{mn}}$ & $114.33^{j}$ & $34.00^{\mathrm{lm}}$ & $3.27^{\text {efghi }}$ & $88.40^{\text {abcd }}$ & $76.56^{\text {cdef }}$ & $228.66^{j}$ & $50.16^{\text {fghij }}$ & $237.73^{\mathrm{e}}$ \\
\hline 24 & SKAU-T-22 & $121.33^{\circ}$ & $95.33^{\mathbf{m}}$ & $25.33^{n}$ & $3.78^{\text {bcd }}$ & $75.80^{\text {bcdefg }}$ & $78.64^{\text {bcde }}$ & $190.66^{\mathbf{m}}$ & $50.66^{\text {defghij }}$ & $201.13^{\mathrm{mn}}$ \\
\hline 25 & SKAU-T-23 & $117.33^{\circ}$ & $91.33^{\mathbf{m}}$ & $24.00^{n}$ & $3.90^{\text {abc }}$ & $79.66^{\text {abcde }}$ & $79.46^{\text {abc }}$ & $182.66^{\mathrm{m}}$ & $53.80^{\text {cde }}$ & $193.60^{\circ}$ \\
\hline 26 & SKAU-T-24 & $135.00^{\mathbf{n}}$ & $103.66^{\mathrm{kl}}$ & $31.33^{\mathrm{m}}$ & $3.64^{\text {cdef }}$ & $74.76^{\text {cdefgh }}$ & $76.76^{\text {cdef }}$ & $207.33^{\mathbf{k l}}$ & $59.46^{\mathrm{a}}$ & $188.20^{p}$ \\
\hline 27 & SKAU-T-25 & $135.33^{n}$ & $107.66^{\mathrm{kl}}$ & $34.33^{\mathrm{klm}}$ & $3.03^{h i j}$ & $87.96^{\text {abcd }}$ & $76.83^{\text {bcdef }}$ & $215.33^{\mathrm{kl}}$ & $49.43^{\text {hijk }}$ & $230.13^{f}$ \\
\hline 28 & SKAU-T-26 & $183.33^{\text {gh }}$ & $147.00^{\mathrm{fg}}$ & $38.00^{\mathbf{i j k l}}$ & $3.88^{\text {abc }}$ & $77.18^{\text {bcdefg }}$ & $80.15^{\text {abc }}$ & $294.00^{\text {gf }}$ & $48.06^{\mathrm{ijk}}$ & $252.23^{d}$ \\
\hline \multicolumn{2}{|c|}{ C.D at $5 \%$} & 12.92 & 6.14 & 5.96 & 0.48 & 16.02 & 4.20 & 12.15 & 3.57 & 5.37 \\
\hline \multicolumn{2}{|c|}{ S.E.Diff } & 6.44 & 3.06 & 2.97 & 0.24 & 7.99 & 2.09 & 6.06 & 1.78 & 2.67 \\
\hline
\end{tabular}


Table.2c Mean performance of Turnip (Brassica rapa var. rapifera) genotypes for various quality traits

\begin{tabular}{|c|c|c|c|c|c|c|c|c|}
\hline S. no & Genotypes & $\begin{array}{c}\text { Total dry } \\
\text { matter (\%) }\end{array}$ & $\begin{array}{c}\text { Total } \\
\text { soluble } \\
\text { solids } \\
\left({ }^{0} \text { Brix }\right)\end{array}$ & $\begin{array}{c}\text { Vit. C } \\
(\mathrm{mg} / 100 \mathrm{~g})\end{array}$ & $\begin{array}{l}\text { Anthocyanin } \\
\text { Content } \\
(\mathrm{mg} / 100 \mathrm{~g})\end{array}$ & $\begin{array}{c}\text { Total } \\
\text { sugars } \\
(\%)\end{array}$ & $\begin{array}{c}\text { Reducing } \\
\text { sugars } \\
(\%)\end{array}$ & $\begin{array}{l}\text { Non-reducing } \\
\text { sugars } \\
(\%)\end{array}$ \\
\hline 1 & SKAU-T-1 & $10.31^{\text {fghi }}$ & $6.80^{\mathrm{a}}$ & $33.00^{\mathrm{a}}$ & $1.73^{\text {no }}$ & $3.74^{\mathrm{a}}$ & $2.70^{\mathrm{a}}$ & $1.04^{\mathrm{bc}}$ \\
\hline 2 & SKAU-T-2 & $7.79^{i}$ & $5.73^{\text {defgh }}$ & $20.66^{\mathrm{kI}}$ & $1.96^{\mathrm{mno}}$ & $2.28^{\text {efghi }}$ & $1.57^{\text {hijk }}$ & $0.69^{\text {bcdefghi }}$ \\
\hline 3 & SKAU-T-3 & $11.28^{\text {defg }}$ & $3.80^{\circ}$ & $24.00^{\text {ghijk }}$ & $3.26^{\mathrm{def}}$ & $3.05^{b}$ & $2.19^{b c}$ & $0.86^{\text {bcdef }}$ \\
\hline 4 & SKAU-T-4 & $9.07^{\mathrm{ghi}}$ & $5.13^{\mathrm{jklm}}$ & $22.00^{\mathbf{i j k}}$ & $2.22^{\operatorname{lm}}$ & $2.43^{\text {defgh }}$ & $1.90^{\text {cdefg }}$ & $0.53^{\text {efghi }}$ \\
\hline 5 & Nageen & $8.64^{\mathrm{hi}}$ & $5.46^{\text {ghijk }}$ & $25.33^{\text {fghi }}$ & $1.73^{\mathrm{no}}$ & $2.36^{\text {defgh }}$ & $1.53^{j k}$ & $0.82^{\text {bcdefgh }}$ \\
\hline 6 & SKAU-T-5 & $11.32^{\text {defg }}$ & $5.46^{\text {ghijk }}$ & $22.66^{\text {hijk }}$ & $3.51^{\mathrm{d}}$ & $2.84^{\text {bed }}$ & $2.03^{\mathrm{cde}}$ & $0.80^{\text {bcdefgh }}$ \\
\hline 7 & SKAU-T-6 & $11.88^{\text {bcdef }}$ & $5.86^{\text {cdefg }}$ & $24.00^{\text {ghijk }}$ & $1.67^{\circ}$ & $1.79^{i}$ & $1.46^{\mathrm{k}}$ & $0.33^{\mathrm{i}}$ \\
\hline 8 & SKAU-T-7 & $13.22^{\text {bcd }}$ & $4.73^{\mathrm{mn}}$ & $32.00^{\mathbf{a b}}$ & $3.14^{\text {efg }}$ & $1.96^{\mathrm{hi}}$ & $1.53^{\mathrm{jk}}$ & $0.42^{\mathrm{hi}}$ \\
\hline 9 & SKAU-T-8 & $11.34^{\text {defg }}$ & $3.66^{\circ}$ & $21.33^{\mathbf{j k l}}$ & $2.87^{\mathrm{ghi}}$ & $2.46^{\text {cdefgh }}$ & $2.00^{\text {cdef }}$ & $0.45^{\text {fghi }}$ \\
\hline 10 & SKAU-T-9 & $10.83^{\text {defgh }}$ & $5.13^{\mathrm{jklm}}$ & $26.33^{\text {efgh }}$ & $1.26^{\mathrm{p}}$ & $2.05^{\text {ghi }}$ & $1.68^{\text {ghijk }}$ & $0.36^{\mathrm{i}}$ \\
\hline 11 & SKAU-T-10 & $12.06^{\text {bcdef }}$ & $6.13^{\mathrm{bcd}}$ & $26.66^{\text {defg }}$ & $1.97^{\mathrm{mno}}$ & $2.63^{\text {bcdef }}$ & $1.92^{\text {cdefg }}$ & $0.84^{\text {bedefg }}$ \\
\hline 12 & SKAU-T-11 & $12.90^{\text {bcde }}$ & $4.86^{\mathrm{klm}}$ & $26.33^{\text {efgh }}$ & $2.90^{\mathrm{ghi}}$ & $3.72^{a}$ & $2.63^{a}$ & $1.09^{\mathbf{a b}}$ \\
\hline 13 & PTWG & $16.14^{\mathrm{a}}$ & $6.73^{a}$ & $28.00^{\text {cdef }}$ & $2.99^{\mathrm{fgh}}$ & $2.42^{\text {cdefgh }}$ & $1.79^{\text {defghij }}$ & $0.63^{\text {cdefghi }}$ \\
\hline 14 & SKAU-T-12 & $10.87^{\text {defgh }}$ & $6.03^{\text {bcde }}$ & $30.33^{\text {abcd }}$ & $3.35^{\mathrm{de}}$ & $2.36^{\text {defgh }}$ & $1.91^{\text {cdefg }}$ & $0.44^{\text {ghi }}$ \\
\hline 15 & SKAU-T-13 & $11.82^{\text {cdef }}$ & $5.96^{\text {cdef }}$ & $28.00^{\text {cdef }}$ & $2.68^{\mathrm{ijk}}$ & $2.25^{\text {efghi }}$ & $1.71^{\text {fghijk }}$ & $0.53^{\text {defghi }}$ \\
\hline 16 & SKAU-T-14 & $14.39^{\mathbf{a b}}$ & $5.66^{\text {efgh }}$ & $28.33^{\text {bcdef }}$ & $1.97^{\mathrm{mn}}$ & $3.80^{\mathrm{a}}$ & $2.70^{\mathrm{a}}$ & $1.10^{\mathbf{a b}}$ \\
\hline 17 & SKAU-T-15 & $13.10^{\text {bed }}$ & $5.03^{\mathrm{klm}}$ & $29.33^{\text {abcde }}$ & $1.81^{\mathrm{no}}$ & $2.63^{\text {bcdef }}$ & $2.19^{b c}$ & $0.44^{\text {ghi }}$ \\
\hline 18 & SKAU-T-16 & $11.95^{\text {bcdef }}$ & $6.26^{b c}$ & $28.33^{\text {bcdef }}$ & $2.42^{\mathbf{k} \mathbf{I}}$ & $2.90^{b c}$ & $1.95^{\text {cdefg }}$ & $0.95^{\text {bed }}$ \\
\hline 19 & SKAU-T-17 & $10.41^{\text {efgh }}$ & $4.56^{n}$ & $23.00^{\mathrm{abc}}$ & $2.87^{\mathrm{ghi}}$ & $2.29^{\text {efghi }}$ & $1.84^{\text {defghi }}$ & $0.44^{\text {fghi }}$ \\
\hline 20 & SKAU-T-18 & $11.56^{\text {cdefg }}$ & $6.46^{\mathbf{a b}}$ & $30.66^{\text {ghijk }}$ & $3.16^{\text {efg }}$ & $2.90^{\mathbf{b c}}$ & $2.02^{\text {cde }}$ & $0.88^{\text {bcde }}$ \\
\hline 21 & SKAU-T-19 & $11.73^{\text {cdef }}$ & $5.30^{\text {hijkl }}$ & $32.00^{\mathbf{a b}}$ & $2.77^{\mathrm{hij}}$ & $2.36^{\text {defgh }}$ & $1.68^{\text {ghijk }}$ & $0.67^{\text {cdefghi }}$ \\
\hline 22 & SKAU-T-20 & $13.97^{\mathrm{abc}}$ & $2.76^{p}$ & $26.00^{\text {efgh }}$ & $4.16^{c}$ & $2.11^{\text {ghi }}$ & $1.66^{\text {ghijk }}$ & $0.45^{\text {fghi }}$ \\
\hline 23 & SKAU-T-21 & $11.46^{\text {cdefg }}$ & $5.60^{\text {efghi }}$ & $18.00^{I}$ & $5.04^{b}$ & $2.21^{\text {fghi }}$ & $1.56^{\text {hijk }}$ & $0.65^{\text {cdefghi }}$ \\
\hline 24 & SKAU-T-22 & $11.36^{\text {defg }}$ & $5.13^{\mathrm{jklm}}$ & $17.66^{1}$ & $2.51^{\mathrm{jkI}}$ & $1.98^{\mathrm{hi}}$ & $1.54^{\mathrm{ijk}}$ & $0.42^{\mathrm{hi}}$ \\
\hline 25 & SKAU-T-23 & $11.59^{\text {cdefg }}$ & $5.56^{\text {fghj }}$ & $21.00^{\mathbf{k I}}$ & $3.05^{\text {fgh }}$ & $2.74^{\text {bcde }}$ & $1.74^{\text {efghijk }}$ & $1.00^{\mathrm{bc}}$ \\
\hline 26 & SKAU-T-24 & $12.73^{\text {bcdef }}$ & $5.20^{\mathrm{ijkl}}$ & $22.00^{\mathbf{i j k}}$ & $5.96^{\mathrm{a}}$ & $3.91^{\mathrm{a}}$ & $2.42^{\mathbf{a b}}$ & $1.48^{\mathrm{a}}$ \\
\hline 27 & SKAU-T-25 & $12.13^{\text {bcdef }}$ & $5.06^{\mathrm{klm}}$ & $25.00^{\text {fghij }}$ & $4.28^{c}$ & $2.89^{b c}$ & $2.06^{\mathrm{cd}}$ & $0.83^{\text {bcdefgh }}$ \\
\hline 28 & SKAU-T-26 & $11.65^{\text {cdef }}$ & $5.56^{\text {fghij }}$ & $22.00^{\mathrm{ijk}}$ & $3.12^{\text {efg }}$ & $2.50^{\text {cdefg }}$ & $1.86^{\text {defgh }}$ & $0.64^{\text {cdefghi }}$ \\
\hline \multicolumn{2}{|c|}{ C.D 5\% } & 2.57 & 0.46 & 3.90 & 0.29 & 0.51 & 0.30 & 0.41 \\
\hline
\end{tabular}




\section{Mean Performance of different Genotypes}

The estimates of mean values (Table $2 a, 2 b$ and $2 \mathrm{c}$ ) revealed that no single genotype was superior for all the characters under study. However SKAU-T-24 $(27.23 \mathrm{~cm})$ followed by SKAU-T-22 $(26.06 \mathrm{~cm})$ and SKAU-T-17 $(25.83 \mathrm{~cm})$ were superior for seedling length. SKAU-T-24 (5.71g) followed by SKAU-T-16 $(5.50 \mathrm{~g})$ and SKAU-T-21 (5.28g) were superior for seedling fresh weight, SKAU-T$24(0.71 \mathrm{~g})$ followed by SKAU-T-20 and SKAU-T-21 (0.66g each) and SKAU-T-2 $(0.23 \mathrm{~g})$ were found superior for seedling dry weight. Nageen $(24.43 \mathrm{~cm})$ followed by SKAU-T-6 $(23.83 \mathrm{~cm})$ and SKAU-T-13 $(23.33 \mathrm{~cm})$ were found superior for leaf length, Nageen $(16.30 \mathrm{~cm})$ followed by SKAU-T-6 $(13.66 \mathrm{~cm})$ and SKAU-T$15(13.40 \mathrm{~cm})$ were found superior for leaf breadth, SKAU-T-7 $(17.05 \mathrm{~cm})$ followed by SKAU-T-23 $(16.40 \mathrm{~cm})$ and SKAU-T-14 $(15.46 \mathrm{~cm})$ for petiole length, SKAU-T-18 $(6.66 \mathrm{~cm})$ followed by SKAU-T-20 $(5.96 \mathrm{~cm})$ and SKAU-T-15 $(5.90 \mathrm{~cm})$ for root length, SKAU-T-7 $(7.93 \mathrm{~cm})$ followed by SKAU-T-3 and SKAU-T-5 $(7.76 \mathrm{~cm}$ each) for root breadth, SKAU-T-14 $(56.43 \mathrm{~cm})$ followed by SKAU-T-7 $(56.06 \mathrm{~cm})$ and SKAU-T-4 $(55.60 \mathrm{~cm})$ for plant length, SKAU-T-20 (11.33) followed by SKAU-T-4 (11) and SKAU-T-2 (10.66) for leaves/plant, Nageen (295g) followed by SKAU-T-4 (285g) and SKAU-T-9 (271.00g) for total plant weight, Nageen $(235.33 \mathrm{~g})$ followed by SKAU-T-4 (231.00g) and SKAU-T-3 (201.16g) for total root weight, SKAU-T-9 (64.66g) followed by PTWG (62.66g) and SKAU-T-3 (61.83) for total shoot weight, SKAU-T-4 (4.29) followed by Nageen (4.17) and SKAU-T-14 (4.12) for root shoot ratio, SKAU-T-19 ( 95.30) followed by Nageen (91.46) and SKAU-T-4 (91.00) for root compactness, SKAU-T-14 (82.96\%) followed by SKAU-T$4(81.03 \%)$ and SKAU-T-23 $(79.46 \%)$ for harvest index, Nageen $(470.66 \mathrm{q} / \mathrm{ha})$ followed by SKAU-T-4 (462.00q/ha) and SKAU-T-3 (402.33q/ha) for root yield/ha, SKAU-T-24 $(59.46 \mathrm{~cm})$ followed by SKAU-T-12 $(58.86 \mathrm{~cm})$ and SKAU-T-5 $(54.76 \mathrm{~cm})$ for plant spread, SKAU-T-6 $\left(287.63 \mathrm{~cm}^{2}\right)$ followed by SKAUT-5 $\left(266.46 \mathrm{~cm}^{2}\right)$ and Nageen $\left(262.33 \mathrm{~cm}^{2}\right)$ for leaf area, PTWG (16.14\%) followed by SKAU-T-14 (14.39\%) and SKAU-T-20 (13.97\%) for total dry matter content, SKAUT-1 (6.80 Brix) followed by PTWG (6.73 Brix) and SKAU-T-18 (6.46 Brix) for total soluble solids, SKAU-T-1 $(33.00 \mathrm{mg} / 100 \mathrm{~g})$ followed by SKAU-T-7 and SKAU-T-19 (32.00 mg/100g each) for Vit. C, SKAU-T-24 $(5.96 \mathrm{mg} / 100 \mathrm{~g})$ followed by SKAU-T-21 $(5.04 \mathrm{mg} / 100 \mathrm{~g})$ and SKAU-T-25 $\quad(4.28$ $\mathrm{mg} / 100 \mathrm{~g}$ ) for anthocyanin content, SKAU-T24 (3.91\%) followed by SKAU-T-14 (3.80\%) and SKAU-T-1 (3.74\%) for total sugars, SKAU-T-14 (2.70\%) and SKAU-T-1 (2.70\%) followed by SKAU-T-11 (2.63 \%) for reducing sugars, and SKAU-T-24 (1.48\%) and SKAU-T-11 (1.09\%) for non-reducing sugars. Similarly a wide range of variation for different traits in turnip was observed by Nasarullah (1994), Khan et al., (2010) in Kale, Santhosha et al., (2015) in cabbage.

The maximum range was recorded for root yield/ha followed by total root weight and total plant weight, while as minimum range was recorded in seedling dry weight followed by non-reducing sugars and reducing sugars.In this study the genotypes showed a wide range of variation for almost all the traits studied. Hence, based on the mean performance of all the genotypes, the genotypes viz., Nageen, SKAU-T-4, SKAUT-3, SKAU-T-9, SKAU-T-14 and PTWG were found superior in terms of root yield/ha and should be used in further breeding programmes.

\section{References}

Anonymous, 2017. Indian Horticulture Data 
Base.

Gomez, K. A and Gomez, A.A. 1983. Statistical procedure for Agricultural research. John Wiley and Sons, New York. 691 p.

Guleira, N. Studies on genetic divergence in temperate radish (Raphanus sativus L.) 2106. [ thesis]. Dr. YS Parmar university of Horticulture and Forestry, Nauni, Solan (HP). p77.

Hammer, K., Gladis, T. H., Laghetti, G. and Pignone, D. 2013. The wild and the grown-remarks on Brassica. International Journal of Agricultural Science 3: 453-480.

Khan, S. H., Ahmad, N., Jabeen, N., Chatto, M. A and Hussain, K. 2010. Biodiversity of Kale (Brassica oleracea var. acephala) in Kashmir valley. The Asian journal of Horticulture, 5(1): 208-210.

Makhdoomi, M. I., Din, G., Ahmad, N., Hussain, K and Gazala, N. 2007. . Genetic variability and selection parameters for yield attributes in radish(Raphanus sativus L. ). Asian Journal of Horticulture. 2(2): 141143.

McCormack JH. Brassica seed production cooperative state research extensive and extensive service; Http://creativecommons.org traces/by nc-nd/2.0/., 2005

Nasarullah. Variability and correlation studies in Turnip (Brassica rapa L.). 1994. [thesis]. Sher-e-Kashmir University of
Agricultural Science and Technology. p71.

Naseeruddin, K. H., Singh, V., Pant, S. C and Rana, D. K. 2011. Genetic Variability and selection parameters for different genotypes of radish (Raphanus sativus L.) under valley condition of Uttrakhand. Progressive Horticulture. 43(2): 256-258.

Persson, K., Falt, A.S and Bothmer, R.V. (2001). Genetic diversity of allozymes in turnip (Brassica rapa var. rapa L. )from the Nordeic area. Heredity. 134: 43-52.

Rakow. G 2004. Species origin and economic importance of Brassica. Biotechnology in Agriculture and Forestry, Vol. 54. New York Springer-Verlag Berlin Heidelberg pp. 3-11.

Santhosha, H. M., Varalakshmi, B. and Manohar, R. K. 2015. Evaluation of early Cauliflower (Brassica rapa var. botrytis L.) germplasm under tropical conditions for various horticultural traits. The bioscan, 10(4): 1631-1635.

Talebi, R., Haghnazari, A and Tabatabaei, I .(2010). Assessment of genetic variation within international collection of Brassica rapa genotypes using inter simple sequence repeat DNA markers. Biharean Biology .4(2): 145-151.

Thamburaj, S and Singh, N. 2018. Textbook of Vegetable, Tuber crops and Spices. ICAR, New Delhi, pp 161

\section{How to cite this article:}

Mir Tabasum Ashraf, Shahnaz Mufti , Khursheed Hussain, Gowhar Ali, Usma jan, Nawaz Ahmad, Iqtab Mushtaq, Afiya Khursheed, Humeera Nazir, Sameena Maqbool and Amreena Sultan. 2021. Comparative Performance of Turnip (Brassica rapa var. rapifera L.) Genotypes for Various Quantitative and Quality Traits. Int.J.Curr.Microbiol.App.Sci. 10(01): 786-793. doi: https://doi.org/10.20546/ijcmas.2021.1001.096 